\title{
Ethnicity sensitive author disambiguation using semi-supervised learning
}

\author{
Gilles Louppe \\ CERN \\ Switzerland
}

\author{
Hussein Al-Natsheh \\ CERN \\ Switzerland
}

\author{
Mateusz Susik \\ CERN \\ Switzerland
}

\author{
Eamonn Maguire \\ CERN \\ Switzerland
}

\begin{abstract}
Author name disambiguation in bibliographic databases is the problem of grouping together scientific publications written by the same person, accounting for potential homonyms and/or synonyms. Among solutions to this problem, digital libraries are increasingly offering tools for authors to manually curate their publications and claim those that are theirs. Indirectly, these tools allow for the inexpensive collection of large annotated training data, which can be further leveraged to build a complementary automated disambiguation system capable of inferring patterns for identifying publications written by the same person. Building on more than 1 million publicly released crowdsourced annotations, we propose an automated author disambiguation solution exploiting this data (i) to learn an accurate classifier for identifying coreferring authors and (ii) to guide the clustering of scientific publications by distinct authors in a semi-supervised way. To the best of our knowledge, our analysis is the first to be carried out on data of this size and coverage. With respect to the state of the art, we validate the general pipeline used in most existing solutions, and improve by: (i) proposing phonetic-based blocking strategies, thereby increasing recall; and (ii) adding strong ethnicity-sensitive features for learning a linkage function, thereby tailoring disambiguation to non-Western author names whenever necessary.
\end{abstract}

\section{Introduction}

In academic digital libraries, author name disambiguation is the problem of grouping together publications written by the same person. Author name disambiguation is often a difficult problem because an author may use different spellings or name variants across their career (synonymy) and/or distinct authors may share the same name (polysemy). Most notably, author disambiguation is often more troublesome for researchers from non-Western cultures, where personal names may be traditionally less diverse (leading to homonym issues) or for which transliteration to Latin characters may not be unique (leading to synonym issues). With the fast growth of the scientific literature, author disambiguation has become a pressing issue since the accuracy of information managed at the level of individuals directly affects: the relevance search of results (e.g., when querying for all publications written by a given author); the reliability of bibliometrics and author rankings (e.g., citation counts or other impact metrics, as studied in (Strotmann and Zhao, 2012)); and/or the relevance of scientific network analysis (Newman, 2001).

Efforts and solutions to author disambiguation have been proposed from various communities (Liu et al. 2014). On the one hand, libraries have maintained authorship control through manual curation, either in a centralized way by hiring professional collaborators or through developing services that invite authors to register their publications themselves 
(e.g., Google Scholar or Inspire-HEP). Recent efforts to create persistent digital identifiers assigned to researchers (e.g., ORCID or ResearcherID), with the objective to embed these identifiers in the submission workflow of publishers or repositories (e.g., Elsevier, arXiv or Inspire-HEP), would univocally solve any disambiguation issue. With the large cost of centralized manual authorship control, or until crowdsourced solutions are more widely adopted, the impact of these efforts are unfortunately limited by the efficiency, motivation and integrity of their active contributors. Similarly, the success of persistent digital identifier efforts is conditioned to a large and ubiquitous adoption by both researchers and publishers. For these reasons, fully automated machine learning-based methods have been proposed during the past decade to provide immediate, less costly, and satisfactory solutions to author disambiguation. In this work, our goal is to explore and demonstrate how both approaches can coexist and benefit from each other. In particular, we study how labeled data obtained through manual curation (either centralized or crowdsourced) can be exploited (i) to learn an accurate classifier for identifying coreferring authors, and (ii) to guide the clustering of scientific publications by distinct authors in a semi-supervised way. Our analysis of parameters and features of this large dataset reveal that the general pipeline commonly used in existing solutions is an effective approach for author disambiguation. Additionally, we propose alternative strategies for blocking based on the phonetization of author names to increase recall. We also propose ethnicity-sensitive features for learning a linkage function, thereby tailoring author disambiguation to non-Western author names whenever necessary.

The remainder of this report is structured as follows. In Section 2, we briefly review machine learning solutions for author disambiguation. The components of our method are then defined in Section 3 and its implementation described in Section 4. Experiments are carried out in Section 5, where we explore and validate features for the supervised learning of a linkage function and compare strategies for the semi-supervised clustering of publications. Finally, conclusions and future works are discussed in Section 6 .

\section{Related work}

As reviewed in (Smalheiser and Torvik, 2009, Ferreira et al., 2012, Levin et al., 2012), author disambiguation algorithms are usually composed of two main components: (i) a linkage function determining whether two publications have been written by the same author; and (ii) a clustering algorithm producing clusters of publications assumed to be written by the same author. Approaches can be classified along several axes, depending on the type and amount of data available, the way the linkage function is learned or defined, or the clustering procedure used to group publications. Methods relying on supervised learning usually make use of a small set of hand-labeled pairs of publications identified as being either from the same or different authors to automatically learn a linkage function between publications (Han et al., 2004, Huang et al., 2006, Culotta et al., 2007, Treeratpituk and Giles, 2009. Tran et al. 2014).

Training data is usually not easily available, therefore unsupervised approaches propose the use of domain-specific, manually designed, linkage functions tailored towards author disambiguation (Malin, 2005; McRae-Spencer and Shadbolt, 2006; Song et al., 2007; Soler. 2007; Kang et al., 2009, Fan et al., 2011, Schulz et al., 2014). These approaches have the advantage of not requiring hand-labeled data, but generally do not perform as well as supervised approaches. To reconcile both worlds, semi-supervised methods make use of small, manually verified, clusters of publications and/or high-precision domain-specific rules to build a training set of pairs of publications, from which a linkage function is then built using supervised learning (Ferreira et al., 2010; Torvik and Smalheiser, 2009, Levin et al., 2012).

Semi-supervised approaches also allow for the tuning of the clustering algorithm when the latter is applied to a mixed set of labeled and unlabeled publications, e.g., by maximizing some clustering performance metric on the known clusters (Levin et al. 2012 ).

Due to the lack of large and publicly available datasets of curated clusters of publications, studies on author disambiguation are usually constrained to validating their results on manually built datasets of limited size and scope (from a few hundred to a few thousand papers, 
Publications

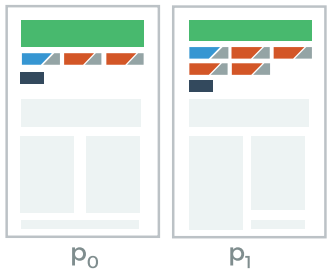

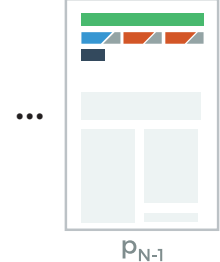

Signatures

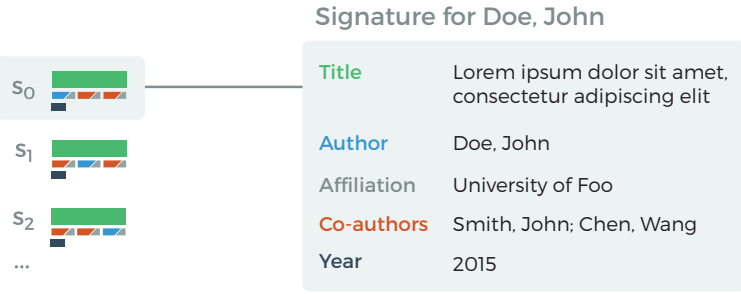

Figure 1: An example signature $s$ for "Doe, John". A signature is defined as unique piece of information identifying an author on a publication, along with any other metadata that can be derived from it, such as publication title, co-authors or date of publication.

with sparse coverage of ambiguous cases), making the true performance of these methods often difficult to assess with high confidence. Additionally, despite devoted efforts to construct them, these datasets are rarely public, making it even more difficult to compare methods using a common benchmark.

In this context, we position the work in this paper as a semi-supervised solution for author disambiguation, with the significant advantage of having a very large collection of more than 1 million crowdsourced annotations of publications whose true authors are identified. The extent and coverage of this data allows us to revisit, validate and nuance previous findings regarding supervised learning of linkage functions, and to better explore strategies for semisupervised clustering. Furthermore, by releasing our data in the public domain, we hope to provide a benchmark on which further research on author disambiguation and related topics can be built.

\section{Semi-supervised author disambiguation}

Formally, let us assume a set of publications $\mathcal{P}=\left\{p_{0}, \ldots, p_{N-1}\right\}$ along with the set of unique individuals $\mathcal{A}=\left\{a_{0}, \ldots, a_{M-1}\right\}$ having together authored all publications in $\mathcal{P}$. Let us define a signature $s \in p$ from a publication as a unique piece of information identifying one of the authors of $p$ (e.g., the author name, his affiliation, along with any other metadata that can be derived from $p$, as illustrated in Figure 1). Let us denote by $\mathcal{S}=\{s \mid s \in p, p \in \mathcal{P}\}$ the set of all signatures that can be extracted from all publications in $\mathcal{P}$.

In this framework, author disambiguation can be stated as the problem of finding a partition $\mathcal{C}=\left\{c_{0}, \ldots, c_{M-1}\right\}$ of $\mathcal{S}$ such that $\mathcal{S}=\cup_{i=0}^{M-1} c_{i}, c_{i} \cap c_{j}=\phi$ for all $i \neq j$, and where subsets $c_{i}$, or clusters, each corresponds to the set of all signatures belonging to the same individual $a_{i}$. Alternatively, the set $\mathcal{A}$ may remain (possibly partially) unknown, such that author disambiguation boils down to finding a partition $\mathcal{C}$ where subsets $c_{i}$ each correspond to the set of all signatures from the same individual (without knowing who). Finally, in the case of partially annotated databases as studied in this work, the set extends with the partial knowledge $\mathcal{C}^{\prime}=\left\{c_{0}^{\prime}, \ldots, c_{M-1}^{\prime}\right\}$ of $\mathcal{C}$, such that $c_{i}^{\prime} \subseteq c_{i}$, where $c_{i}^{\prime}$ may be empty. Or put otherwise, the set extends with the assumption that all signatures $s \in c_{i}^{\prime}$ belong to the same author.

Inspired by several previous works described in Section 2, we cast in this work author disambiguation into a semi-supervised clustering problem. Our algorithm is composed of three parts, as sketched in Figure 2 (i) a blocking scheme whose goal is to roughly precluster signatures $\mathcal{S}$ into smaller groups in order to reduce computational complexity; (ii) the construction of a linkage function $d$ between signatures using supervised learning; and (iii) the semi-supervised clustering of all signatures within the same block, using $d$ as a pseudo distance metric. 


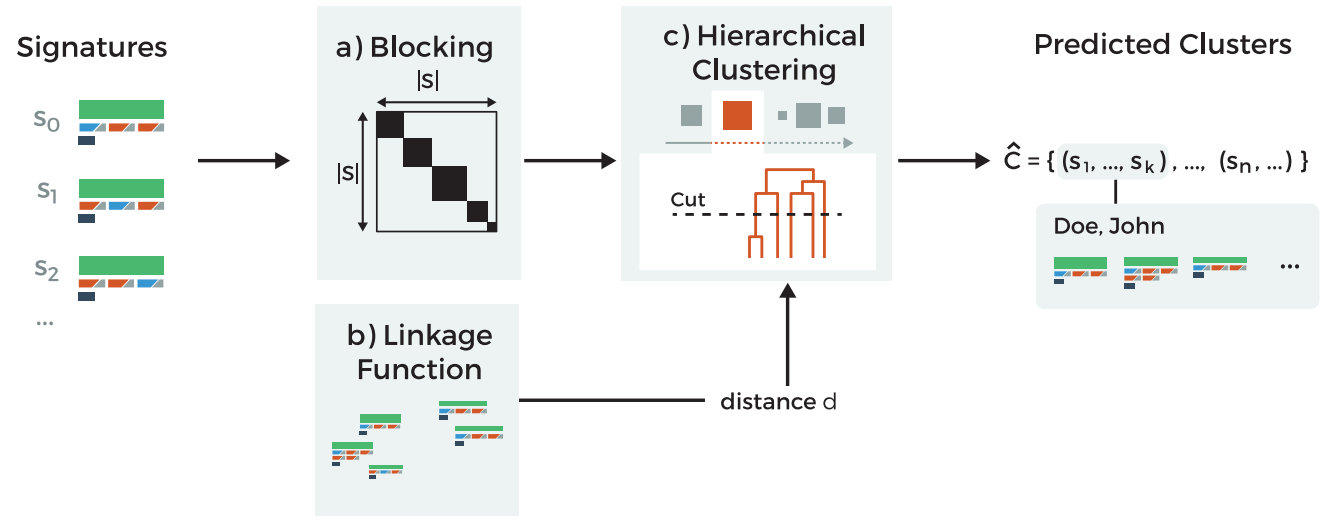

Figure 2: Pipeline for author disambiguation: (a) signatures are blocked to reduce computational complexity, (b) a linkage function is built with supervised learning, (c) independently within each block, signatures are grouped using hierarchical agglomerative clustering.

\subsection{Blocking}

As in previous works, the first part of our algorithm consists of dividing signatures $\mathcal{S}$ into disjoint subsets $\mathcal{S}_{b_{0}}, \ldots, \mathcal{S}_{b_{K-1}}$, or blocks (Fellegi and Sunter, 1969), followed by carrying out author disambiguation on each one of these blocks independently. By doing so, the computational complexity of clustering (see Section 3.3 typically reduces from $O\left(|\mathcal{S}|^{2}\right)$ to $O\left(\sum_{b}\left|\mathcal{S}_{b}\right|^{2}\right)$, which is much more tractable as the number of signatures increases. Since disambiguation is performed independently per block, a good blocking strategy should be designed such that signatures from the same author are all mapped to the same block, otherwise their correct clustering would not be possible in later stages of the workflow. As a result, blocking should be a balance between reduced complexity and maximum recall.

The simplest and most common strategy for blocking, referred to hereon in as Surname and First Initial (SFI), groups signatures together if they share the same surname(s) and the same first given name initial (e.g., SFI("Doe, John") == "Doe, J"). Despite satisfactory performance, there are several cases where this simple strategy fails to cluster related pairs of signatures together, including:

1. There are different ways of writing an author name, or signatures contain a typo (e.g., "Mueller, R." and "Muller, R.", "Tchaikovsky, P." and "Czajkowski, P.").

2. An author has multiple surnames and some signatures place the first part of the surname within the given names (e.g., "Martinez Torres, A." and "Torres, A. Martinez").

3. An author has multiple surnames and, on some signatures, only the first surname is present (e.g., "Smith-Jones, A." and "Smith, A.")

4. An author has multiple given names and they are not always all recorded (e.g., "Smith, Jack" and "Smith, A. J.")

5. An authors surname changed (e.g., due to marriage).

To account for these issues we propose instead to block signatures based on the phonetic representation of the normalized surname. Normalization involves stripping accents (e.g., "Jabłoński, E" $\rightarrow$ "Jablonski, L") and name affixes that inconsistently appear in signatures (e.g., "van der Waals, J. D." $\rightarrow$ "Waals, J. D."), while phonetization is based either on the Double Metaphone (Philips, 2000), the NYSIIS (Taft, 1970) or the Soundex (The National Archives, 2007) phonetic algorithms for mapping author names to their pronunciations. Together, these processing steps allow for grouping of most name variants of the same person in the same block with a small increase in the overall computational complexity, thereby solving case 1 . 
In the case of multiple surnames (cases 2 and 3), we propose to block signatures in two phases. In the first phase, all the signatures with a single surname are clustered together. Every different surname token creates a new block. In the second phase, the signatures with multiple surnames are compared with the blocks for the first and last surname. If the first surnames of an author were already used as the last given names on some of the signatures, the new signature is assigned to the block of the last surname (case 2). Otherwise, the signature is assigned to the block of the first surname (case 3). Finally, to prevent the creation of too large blocks, signatures are further divided along their first given name initial. Cases 4 and 5 are not explicitly handled.

\subsection{Linkage function}

Supervised classification. The second part of the algorithm is the automatic construction of a pair-wise linkage function between signatures for use during the clustering step which groups all signatures from the same author.

Formally, the goal is to build a function $d: \mathcal{S} \times \mathcal{S} \mapsto[0,1]$, such that $d\left(s_{1}, s_{2}\right)$ approaches 0 if both signatures $s_{1}$ and $s_{2}$ belong to the same author, and 1 otherwise. This problem can be cast as a standard supervised classification task, where inputs are pairs of signatures and outputs are classes 0 (same authors), and 1 (distinct authors). In this work, we evaluate Random Forests (RF, Breiman (2001)), Gradient Boosted Regression Trees (GBRT, Friedman (2001)), and Logistic Regression (Fan et al., 2008) as classifiers.

Input features. In most cases, supervised learning algorithms assume the input space $\mathcal{X}$ to be numeric $\left(e . g ., \mathbb{R}^{p}\right)$, making them not directly applicable to structured input spaces such as $\mathcal{S} \times \mathcal{S}$. Following previous works, pairs of signatures $\left(s_{1}, s_{2}\right)$ are first transformed to vectors $v \in \mathbb{R}^{p}$ by building so-called similarity profiles (Treeratpituk and Giles, 2009) on which supervised learning is carried out. In this work, we design and evaluate fifteen standard input features based on the comparison of signature fields, as reported in the first half of Table 1. As an illustrative example, the Full name feature corresponds to the similarity between the (full) author name fields of the two signatures, as measured using as combination operator the cosine similarity between their respective $(n, m)-T F-I D F$ vector representation: ${ }^{1}$. Similarly, the Year difference feature measures the absolute difference between the publication date of the articles to which the two signatures respectively belong.

Author names from different cultures, origins or ethnic groups are likely to be disambiguated using different strategies (e.g., pairs of signatures with French author names versus pairs of signatures with Chinese author names) (Treeratpituk and Giles, 2012, Chin et al., 2014). To support our disambiguation algorithm, we added seven features to our feature set, with each evaluating the degree of belonging of both signatures to an ethnic group, as reported in the second half of Table 1 .

More specifically, using census data extracted from (Ruggles et al. 2008), we build a support vector machine classifier (using a linear kernel and one-versus-all classification scheme) for mapping the $(1,5)$-TF-IDF representation of an author name to one of the seven ethnic groups. Given a pair of signatures $\left(s_{1}, s_{2}\right)$, the proposed ethnicity features are each computed as the estimated probability of $s_{1}$ belonging to the corresponding ethnic group, multiplied by the estimated probability of $s_{2}$ belonging to the same group. In doing so, the expectation is for the linkage function to become sensitive to the actual origin of the authors depending on the values of these features. Indirectly, let us also note that these features hold discriminative power since if author names are predicted to belong to different ethnic groups, then they are also likely to correspond to distinct people.

Building a training set. The distinctive aspect of our work is the knowledge of more than 1 million crowdsourced annotations $\mathcal{C}^{\prime}=\left\{c_{0}^{\prime}, \ldots, c_{M-1}^{\prime}\right\}$, indicating together that all signature $s \in c_{i}^{\prime}$ are known to correspond to the same individual $a_{i}$. In particular, this data can be used to generate positive pairs $\left(x=\left(s_{1}, s_{2}\right), y=0\right)$ for all $s_{1}, s_{2} \in c_{i}^{\prime}$, for all $i$. Similarly, negative pairs $\left(x=\left(s_{1}, s_{2}\right), y=1\right)$ can be extracted for all $s_{1} \in c_{i}^{\prime}, s_{2} \in c_{j}^{\prime}$, for all $i \neq j$.

\footnotetext{
${ }^{1}(n, m)$ denotes that the $T F-I D F$ vectors are computed from character $n, n+1, \ldots, m$-grams. When not specified, $T F-I D F$ vectors are otherwise computed from words.
} 
Table 1: Input features for learning a linkage function

\begin{tabular}{|l|l|}
\hline Feature & Combination operator \\
\hline \hline Full name & Cosine similarity of $(2,4)-$ TF-IDF \\
Given names & Cosine similarity of $(2,4)$-TF-IDF \\
First given name & Jaro-Winkler distance \\
Gecond given name & Jaro-Winkler distance \\
Affiliation & Equality \\
Co-authors & Cosine similarity of $(2,4)-T F-I D F$ \\
Title & Cosine similarity of TF-IDF \\
Journal & Cosine similarity of $(2,4)-T F-I D F$ \\
Abstract & Cosine similarity of (2, 4)-TF-IDF \\
Keywords & Cosine similarity of TF-IDF \\
Collaborations & Cosine similarity of TF-IDF \\
References & Cosine similarity of TF-IDF \\
Subject & Cosine similarity of TF-IDF \\
Year difference & Cosine similarity of TF-IDF \\
White & Absolute difference \\
Black & Product of estimated probabilities \\
American Indian or Alaska Native & Product of estimated probabilities \\
Chinese & Product of estimated probabilities \\
Japanese & Product of estimated probabilities \\
Other Asian or Pacific Islander & Product of estimated probabilities \\
Others & Product of estimated probabilities \\
\hline & Product of estimated probabilities \\
\hline
\end{tabular}

The most straightforward approach for building a training set on which to learn a linkage function is to sample an equal number of positive and negative pairs, as suggested above. By observing that the linkage function $d$ will eventually be used only on pairs of signatures from the same block $S_{b}$, a further refinement for building a training set is to restrict positive and negative pairs $\left(s_{1}, s_{2}\right)$ to only those for which $s_{1}$ and $s_{2}$ belong to the same block. In doing so, the trained classifier is forced to learn intra-block discriminative patterns rather than inter-block differences. Furthermore, as noted in (Lange and Naumann, 2011), most signature pairs are non-ambiguous: if both signatures share the same author names, then they correspond to the same individual, otherwise they do not. Rather than sampling pairs uniformly at random, we propose to oversample difficult cases when building the training set (i.e., pairs of signatures with different author names corresponding to same individual, and pairs of signatures with identical author names but corresponding to distinct individuals) in order to improve the overall accuracy of the linkage function.

\subsection{Semi-supervised clustering}

The last component of our author disambiguation pipeline is clustering, that is the process of grouping together, within a block, all signatures from the same individual (and only those). As for many other works on author disambiguation, we make use of hierarchical clustering (Ward Jr, 1963) for building clusters of signatures in a bottom-up fashion. The method involves iteratively merging together the two most similar clusters until all clusters are merged together at the top of the hierarchy. Similarity between clusters is evaluated using either complete, single or average linkage, using as a pseudo-distance metric the probability that $s_{1}$ and $s_{2}$ correspond to distinct authors, as calculated from the custom linkage function $d$ from Section 3.2

To form flat clusters from the hierarchy, one must decide on a maximum distance threshold above which clusters are considered to correspond to distinct authors. Let us denote by $\mathcal{S}^{\prime}=\left\{s \mid s \in c^{\prime}, c^{\prime} \in \mathcal{C}^{\prime}\right\}$ the set of all signatures for which partial clusters are known. Let us also denote by $\widehat{\mathcal{C}}$ the predicted clusters for all signatures in $\mathcal{S}$, and by $\widehat{\mathcal{C}}^{\prime}=\left\{\widehat{c} \cap \mathcal{S}^{\prime} \mid \widehat{c} \in \widehat{\mathcal{C}}\right\}$ the predicted clusters restricted to signatures for which partial clusters are known. From these, we evaluate the following semi-supervised cut-off strategies, as illustrated in Figure 3 . 


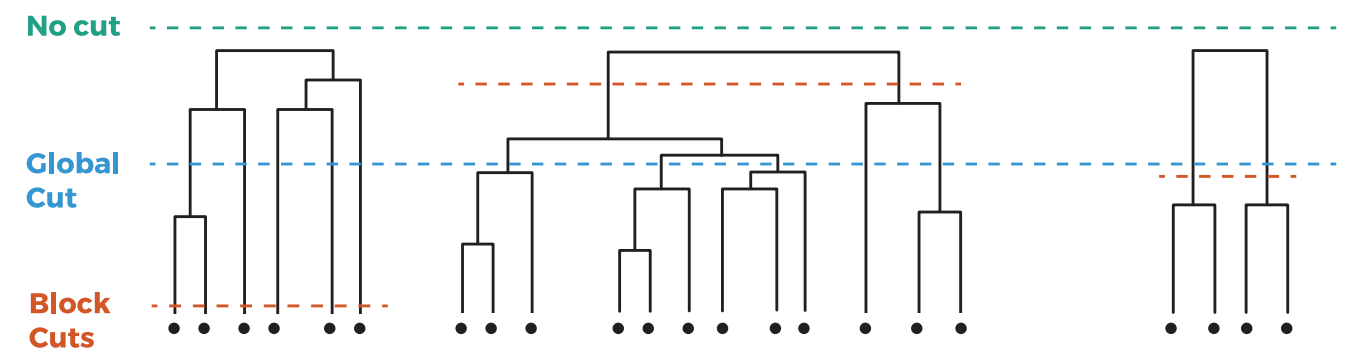

Figure 3: Semi-supervised cut-off strategies to form flat clusters of signatures.

- No cut: all signatures from the same block are assumed to be from the same author.

- Global cut: the threshold is chosen globally over all blocks, as the one maximizing some score $f\left(\mathcal{C}^{\prime}, \widehat{\mathcal{C}}^{\prime}\right)$.

- Block cut: the threshold is chosen locally at each block $b$, as the one maximizing some score $f\left(\mathcal{C}_{b}^{\prime}, \widehat{\mathcal{C}}_{b}^{\prime}\right)$. In case $\mathcal{C}_{b}^{\prime}$ is empty, then all signatures from $b$ are clustered together.

\section{Implementation}

As part of this work, we developed a stand-alone application for author disambiguation, publicly available onlin $\AA^{2}$ for free reuse or study. Our implementation builds upon the Python scientific stack, making use of the Scikit-Learn library (Pedregosa et al., 2011) for the supervised learning of a linkage function and of SciPy (Jones et al., 01 ) for clustering. All components of the disambiguation pipeline have been designed to follow the ScikitLearn API (Buitinck et al. 2013), making them easy to maintain, understand and reuse. Our implementation is made to be efficient, exploiting parallelization when available, and ready for production environments. It is also designed to be runnable in an incremental fashion, by enabling disambiguation only on specified blocks if desired, instead of having to run the disambiguation process on the whole signature set.

\section{Experiments}

\subsection{Data}

The author disambiguation solution proposed in this work, along with its enhancements, are evaluated on data extracted from the INSPIRE portal (Gentil-Beccot et al., 2009), a digital library for scientific literature in high-energy physics. Overall, the portal holds more than 1 million publications $\mathcal{P}$, forming in total a set $\mathcal{S}$ of more than 10 million signatures. Out of these, around $13 \%$ have been claimed by their original authors, marked as such by professional curators or automatically assigned to their true authors thanks to persistent identifiers provided by publishers or other sources. Together, they constitute a trusted set $\left(\mathcal{S}^{\prime}, \mathcal{C}^{\prime}\right)$ of 15388 distinct individuals sharing 36340 unique author names spread within 1201763 signatures on 360066 publications. This data covers several decades in time and dozens of author nationalities worldwide.

Following the INSPIRE terms of use, the signatures $\mathcal{S}^{\prime}$ and their corresponding clusters $\mathcal{C}^{\prime}$ are released online ${ }^{3}$ under the $\mathrm{CC} 0$ license. To the best of our knowledge, data of this size and coverage is the first to be publicly released in the scope of author disambiguation research.

${ }^{2}$ https://github.com/glouppe/beard

$3^{3}$ https://github.com/glouppe/paper-author-disambiguation 


\subsection{Evaluation protocol}

Experiments carried out to study the impact of the proposed algorithmic components and refinements, as described in Section 3, follow a standard 3-fold cross-validation protocol, using $\left(\mathcal{S}^{\prime}, \mathcal{C}^{\prime}\right)$ as ground-truth dataset. To replicate the $\left|\mathcal{S}^{\prime}\right| /|\mathcal{S}| \approx 13 \%$ ratio of claimed signatures with respect to the total set of signatures, as on the INSPIRE platform, crossvalidation folds are constructed by sampling $13 \%$ of claimed signatures to form a training set $\mathcal{S}_{\text {train }}^{\prime} \subseteq \mathcal{S}^{\prime}$. The remaining signatures $\mathcal{S}_{\text {test }}^{\prime}=\mathcal{S}^{\prime} \backslash \mathcal{S}_{\text {train }}^{\prime}$ are used for testing. Therefore, $\mathcal{C}_{\text {train }}^{\prime}=\left\{c^{\prime} \cap \mathcal{S}_{\text {train }}^{\prime} \mid c^{\prime} \in \mathcal{C}^{\prime}\right\}$ represents the partial known clusters on the training fold, while $\mathcal{C}_{\text {test }}^{\prime}$ are those used for testing.

As commonly performed in author disambiguation research, we evaluate the predicted clusters over testing data $\mathcal{C}_{\text {test }}^{\prime}$, using both B3 and pairwise precision, recall and F-measure, as defined below:

$$
\begin{aligned}
P_{\mathrm{B} 3}(\mathcal{C}, \widehat{\mathcal{C}}, \mathcal{S}) & =\frac{1}{|\mathcal{S}|} \sum_{s \in \mathcal{S}} \frac{|c(s) \cap \widehat{c}(s)|}{|\widehat{c}(s)|} \\
R_{\mathrm{B} 3}(\mathcal{C}, \widehat{\mathcal{C}}, \mathcal{S}) & =\frac{1}{|\mathcal{S}|} \sum_{s \in \mathcal{S}} \frac{|c(s) \cap \widehat{\mathcal{C}}(s)|}{|c(s)|} \\
F_{\mathrm{B} 3}(\mathcal{C}, \widehat{\mathcal{C}}, \mathcal{S}) & =\frac{2 P_{\mathrm{B} 3}(\mathcal{C}, \widehat{\mathcal{C}}, \mathcal{S}) R_{\mathrm{B} 3}(\mathcal{C}, \widehat{\mathcal{C}}, \mathcal{S})}{P_{\mathrm{B} 3}(\mathcal{C}, \widehat{\mathcal{C}}, \mathcal{S})+P_{\mathrm{B} 3}(\mathcal{C}, \widehat{\mathcal{C}}, \mathcal{S})} \\
P_{\text {pairwise }}(\mathcal{C}, \widehat{\mathcal{C}}) & =\frac{|p(\mathcal{C}) \cap p(\widehat{\mathcal{C}})|}{|p(\widehat{\mathcal{C}})|} \\
R_{\text {pairwise }}(\mathcal{C}, \widehat{\mathcal{C}}) & =\frac{|p(\mathcal{C}) \cap p(\widehat{\mathcal{C}})|}{|p(\mathcal{C})|} \\
F_{\text {pairwise }}(\mathcal{C}, \widehat{\mathcal{C}}) & =\frac{2 P_{\text {pairwise }}(\mathcal{C}, \widehat{\mathcal{C}}) R_{\text {pairwise }}(\mathcal{C}, \widehat{\mathcal{C}})}{P_{\text {pairwise }}(\mathcal{C}, \widehat{\mathcal{C}})+R_{\text {pairwise }}(\mathcal{C}, \widehat{\mathcal{C}})}
\end{aligned}
$$

and where $c(s)$ (resp. $\widehat{c}(s))$ is the cluster $c \in \mathcal{C}$ such that $s \in c$ (resp. the cluster $\widehat{c} \in \widehat{\mathcal{C}}$ such that $s \in \widehat{c})$, and where $p(\mathcal{C})=\cup_{c \in \mathcal{C}}\left\{\left(s_{1}, s_{2}\right) \mid s_{1}, s_{2} \in c, s_{1} \neq s_{2}\right\}$ is the set of all pairs of signatures from the same clusters in $\mathcal{C}$. Precision evaluates whether signatures are grouped only with signatures from the same true clusters, while recall measures the extent to which all signatures from the same true clusters are effectively grouped together. The F-measure is the harmonic mean between these two quantities. In the analysis below, we rely primarily on the B3 F-measure for discussing results, as the pairwise variant tends to favor large clusters (because the number of pairs is quadratic with the cluster size), hence unfairly giving preference to authors with many publications. By contrast, the B3 F-measure weights clusters linearly with respect to their size. General conclusions drawn below remain however consistent for pairwise F.

\subsection{Results and discussion}

Baseline. Results presented in Table 2 are discussed with respect to a baseline solution using the following combination of components:

- Blocking: same surname and the same first given name initial strategy (SFI);

- Linkage function: all 22 features defined in Table1, gradient boosted regression trees as supervised learning algorithm and a training set of pairs built from $\left(\mathcal{S}_{\text {train }}^{\prime}, \mathcal{C}_{\text {train }}^{\prime}\right)$, by balancing easy and difficult cases.

- Clustering: agglomerative clustering using average linkage and block cuts found to maximize $F_{\mathrm{B} 3}\left(\mathcal{C}_{\text {train }}^{\prime}, \widehat{\mathcal{C}}_{\text {train }}^{\prime}, \mathcal{S}_{\text {train }}^{\prime}\right)$.

Blocking. The good precision of the baseline (0.9901), but its lower recall (0.9760) suggest that the blocking strategy might be the limiting factor to further overall improvements. As shown in Table 3 , the maximum recall (i.e., if within a block, all signatures were clustered 
Table 2: Average precision, recall and f-measure scores on test folds.

\begin{tabular}{|l|ccc|ccc|}
\hline & \multicolumn{4}{|c|}{ B3 } & \multicolumn{3}{c|}{ Pairwise } \\
Description & $P$ & $R$ & $F$ & $P$ & $R$ & $F$ \\
\hline \hline Baseline & 0.9901 & 0.9760 & 0.9830 & 0.9948 & 0.9738 & 0.9842 \\
\hline Blocking = SFI & 0.9901 & 0.9760 & 0.9830 & 0.9948 & 0.9738 & 0.9842 \\
Blocking = Double metaphone & 0.9856 & 0.9827 & 0.9841 & 0.9927 & 0.9817 & 0.9871 \\
Blocking = NYSIIS & 0.9875 & 0.9826 & $\mathbf{0 . 9 8 5 0}$ & 0.9936 & 0.9814 & $\mathbf{0 . 9 8 7 5}$ \\
Blocking = Soundex & 0.9886 & 0.9745 & 0.9815 & 0.9935 & 0.9725 & 0.9828 \\
\hline No name normalization & 0.9887 & 0.9697 & 0.9791 & 0.9931 & 0.9658 & 0.9793 \\
Name normalization & 0.9901 & 0.9760 & $\mathbf{0 . 9 8 3 0}$ & 0.9948 & 0.9738 & $\mathbf{0 . 9 8 4 2}$ \\
\hline Classifier = GBRT & 0.9901 & 0.9760 & 0.9830 & 0.9948 & 0.9738 & 0.9842 \\
Classifier = Random Forests & 0.9909 & 0.9783 & $\mathbf{0 . 9 8 4 6}$ & 0.9957 & 0.9752 & $\mathbf{0 . 9 8 5 4}$ \\
Classifier = Linear Regression & 0.9749 & 0.9584 & 0.9666 & 0.9717 & 0.9569 & 0.9643 \\
\hline Training pairs = Non-blocked, uniform & 0.9793 & 0.9630 & 0.9711 & 0.9756 & 0.9629 & 0.9692 \\
Training pairs = Blocked, uniform & 0.9854 & 0.9720 & 0.9786 & 0.9850 & 0.9707 & 0.9778 \\
Training pairs = Blocked, balanced & 0.9901 & 0.9760 & $\mathbf{0 . 9 8 3 0}$ & 0.9948 & 0.9738 & $\mathbf{0 . 9 8 4 2}$ \\
\hline Clustering = Average linkage & 0.9901 & 0.9760 & $\mathbf{0 . 9 8 3 0}$ & 0.9948 & 0.9738 & $\mathbf{0 . 9 8 4 2}$ \\
Clustering = Single linkage & 0.9741 & 0.9603 & 0.9671 & 0.9543 & 0.9626 & 0.9584 \\
Clustering = Complete linkage & 0.9862 & 0.9709 & 0.9785 & 0.9920 & 0.9688 & 0.9803 \\
\hline No cut & 0.9024 & 0.9828 & 0.9409 & 0.8298 & 0.9776 & 0.8977 \\
Global cut & 0.9892 & 0.9737 & 0.9814 & 0.9940 & 0.9727 & 0.9832 \\
Block cut & 0.9901 & 0.9760 & $\mathbf{0 . 9 8 3 0}$ & 0.9948 & 0.9738 & $\mathbf{0 . 9 8 4 2}$ \\
\hline Combined best settings & 0.9888 & 0.9848 & $\mathbf{0 . 9 8 6 8}$ & 0.9951 & 0.9831 & $\mathbf{0 . 9 8 9 0}$ \\
\hline
\end{tabular}

Table 3: Maximum recall $R_{\mathrm{B} 3}^{*}$ and $R_{\text {pairwise }}^{*}$ of blocking strategies, and their number of blocks on $\mathcal{S}^{\prime}$.

\begin{tabular}{|l|cc|c|}
\hline Blocking & $R_{\mathrm{B} 3}^{*}$ & $R_{\text {pairwise }}^{*}$ & \# blocks \\
\hline \hline SFI & 0.9828 & 0.9776 & 12978 \\
Double metaphone & 0.9907 & 0.9863 & 9753 \\
NYSIIS & 0.9902 & 0.9861 & 10857 \\
Soundex & 0.9906 & 0.9863 & 9403 \\
\hline
\end{tabular}

optimally) for SFI is 0.9828 . At the price of fewer and therefore slightly larger blocks (as reported in the right column of Table 3), the proposed phonetic-based blocking strategies show better maximum recall (all around 0.9905), thereby pushing further the upper bound on the maximum performance of author disambiguation. Let us remind however that the reported maximum recalls for the blocking strategies using phonetization are also raised due to the better handling of multiple surnames, as described in Section 3.1.

As Table 2 shows, switching to either Double metaphone or NYSIIS phonetic-based blocking allows to improve the overall F-measure score, trading precision for recall. In particular, the NYSIIS-based phonetic blocking shows to be the most effective when applied to the baseline (with an F-measure of 0.9850) while also being the most efficient computationally (with 10857 blocks versus 12978 for the baseline).

Finally, let us also note that Table 3 corroborates the estimation of (Torvik and Smalheiser, 2009 ), stating that SFI blocking has a recall around $98 \%$ on real data.

Name normalization. As discussed previously, the seemingly insignificant step of normalizing author names (stripping accents, removing affixes), as performed in the baseline, is shown to be important. Results from Table 2 clearly suggest that not normalizing significantly reduces performance (yielding an F-measure of 0.9830 when normalizing, but decreasing to 0.9791 when raw author name strings are used instead).

Linkage function. Let us first comment on the results regarding the supervised algorithm used to learn the linkage function. As Table 2 indicates, both tree-based algorithms appear to be significantly better fit than Linear Regression (0.9830 and 0.9846 for GBRT and Random 
Forests versus 0.9666 for Linear Regression). This result is consistent with (Treeratpituk and Giles, 2009) which evaluated the use of Random Forests for author disambiguation, but contradicts results of (Levin et al. 2012) for which Logistic Regression appeared to be the best classifier. Provided hyper-parameters are properly tuned, the superiority of tree-based methods is in our opinion not surprising. Indeed, given the fact that the optimal linkage function is likely to be non-linear, non-parametric methods are expected to yield better results, as the experiments here confirm.

Second, properly constructing a training set of positive and negative pairs of signatures from which to learn a linkage function yields a significant improvement. A random sampling of positive and negative pairs, without taking blocking into account, significantly impacts the overall performance (0.9711). When pairs are drawn only from blocks, performance increases (0.9786), which confirms our intuition that $d$ should be built only from pairs it will be used to eventually cluster. Finally, making the classification problem more difficult by oversampling complex cases proves to be relevant, by further improving the disambiguation results $(0.9830)$.

Using Recursive Feature Elimination Guyon et al. (2002), we next evaluate the usefulness of all fifteen standard and seven additional ethnicity features for learning the linkage function. The analysis consists in using the baseline algorithm first using all twenty two features, to determine the least discriminative from feature importances (Louppe et al., 2013), and then re-learn the baseline using all but that one feature. That process is repeated recursively until eventually only one feature remains. Results are presented in Figure 4 for one of the three folds, starting from the far right with the baseline and Second given name being the least important feature, and ending on the left with all features eliminated but Chinese. As the figure illustrates, the most important features are ethnic-based features (Chinese, Other Asian, Black) along with Co-authors, Affiliation and Full name. Adding the remaining other features only brings marginal improvements, with Journal, Abstract, Collaborations, References, Given name initial and Second given name being almost insignificant. Overall, these results highlight the added value of the proposed ethnicity features. Their duality in modeling both the similarity between author names and their origins make them very strong predictors for author disambiguation. The results also corroborate those from (Kang et al. 2009) or (Ferreira et al., 2010), who found that the similarity between co-authors was a highly discriminative feature. If computational complexity is a concern, this analysis also shows how decent performance can be achieved using only a very small set of features, as also observed in (Treeratpituk and Giles, 2009) or (Levin et al., 2012).

Semi-supervised clustering. The last part of our experiment concerns the study of agglomerative clustering and the best way to find a cut-off threshold to form clusters. Results from Table 2 first clearly indicate that average linkage is significantly better than both single and complete linkage.

Clustering together all signatures from the same block is the least effective strategy (0.9409), but yields anyhow surprisingly decent accuracy, given the fact it requires almost no computation (i.e., both learning a linkage function and running agglomerative clustering can be skipped - only the blocking function is needed to group signatures). In particular, this result reveals that author names are not ambiguous in most cases ${ }^{4}$ and that only a small fraction of them requires advanced disambiguation procedures. On the other hand, both global and block cut thresholding strategies give very good results, with a slight advantage for block cuts $(0.9814$ versus 0.9830$)$, as expected. In case $\mathcal{S}_{b}^{\prime}$ is empty (e.g., because it corresponds to a young researcher at the beginning of his career), this therefore suggests that either using a cut-off threshold learned globally from the known data or using SFI would in general give satisfactory results, only marginally worse than if claimed signatures had been known.

Combined best settings. When all best settings are combined (i.e., Blocking = NYSIIS, Name normalization, Classifier $=$ Random Forests, Training pairs $=$ blocked and balanced, Clustering $=$ Average linkage, Block cuts), performance reaches 0.9862 , i.e., the best of all

\footnotetext{
${ }^{4}$ This holds for the data we extracted, but may in the future, with the rise of non-Western researchers, be an underestimate of the ambiguous cases.
} 
Figure 4: Recursive Feature Elimination analysis.

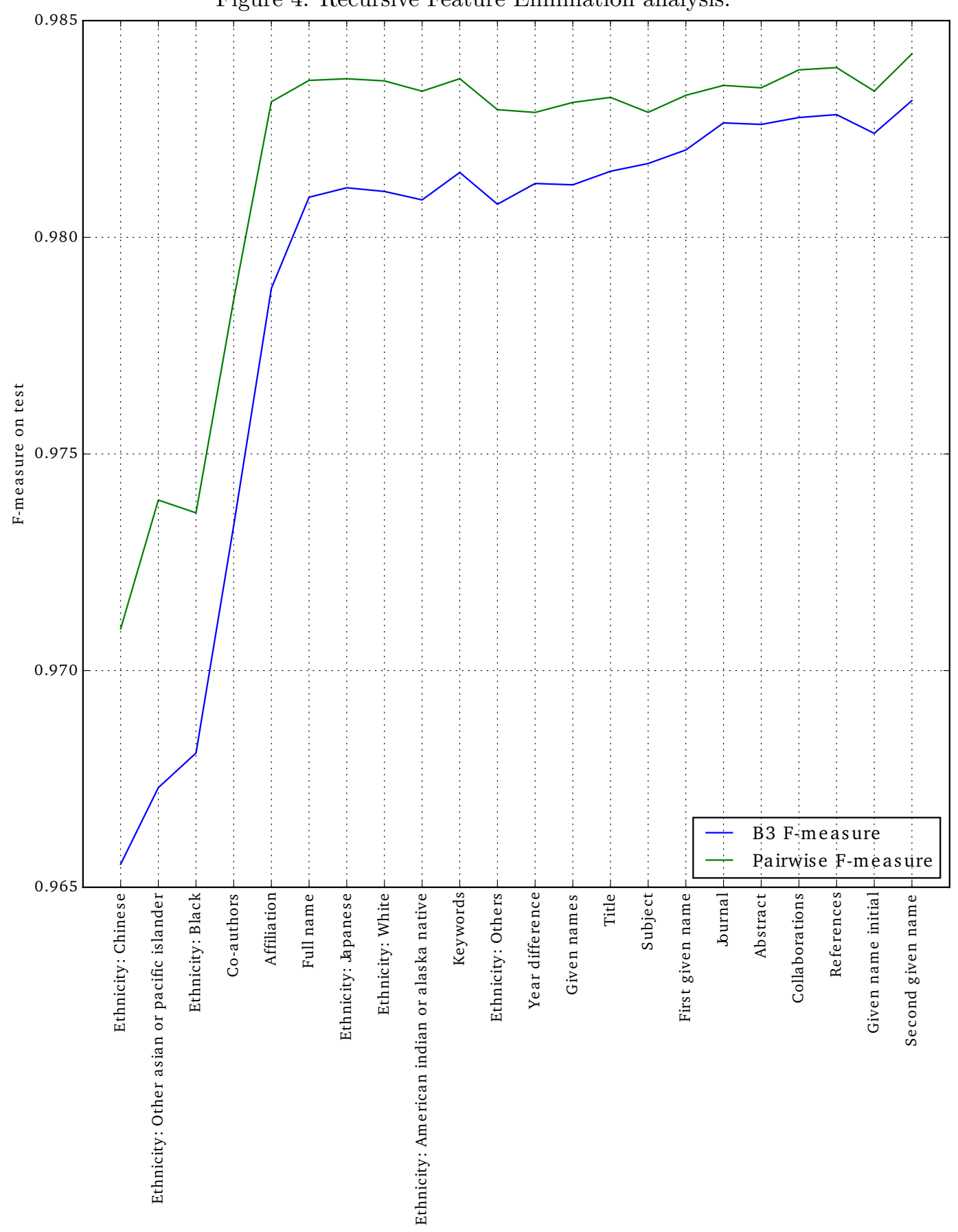


reported results. In particular, this combination exhibits both the high recall of phonetic blocking based on the NYSIIS algorithm and the high precision of Random Forests.

\section{Conclusions}

In this work, we have revisited and validated the general author disambiguation pipeline introduced in previous independent research work. The generic approach is composed of three components, whose design and tuning are all critical to good performance: (i) a blocking function for pre-clustering signatures and reducing computational complexity, (ii) a linkage function for identifying signatures with coreferring authors and (iii) the agglomerative clustering of signatures. Making use of a distinctively large dataset of more than 1 million crowdsourced annotations, we experimentally study all three components and propose further improvements. With regards to blocking, we suggest to use phonetization of author names to increase recall while maintaining low computational complexity. For the linkage function, we introduce ethnicity-sensitive features for the automatic tailoring of disambiguation to non-Western author names whenever necessary. Finally, we explore semi-supervised cut-off threshold strategies for agglomerative clustering. For all three components, experiments show that our refinements all yield significantly better author disambiguation accuracy.

Overall, these results all encourage further improvements and research. For blocking, one of the open challenges is to manage signatures with inconsistent surnames or inconsistent first given names (cases 4 and 5, as described in Section 3.1) while maintaining blocks to a tractable size. As phonetic algorithms are not yet perfect, another direction for further work is the design of better phonetization functions, tailored for author disambiguation. For the linkage function, the good results of the proposed features pave the way for further research in ethnicity-sensitive author disambiguation. The automatic fitting of the pipeline to cultures and ethnic groups for which standard author disambiguation is known to be less efficient (e.g., Chinese authors with many homonyms) indeed constitutes a direction of research with great potential benefits for the concerned scientific communities.

As part of this study, we also publicly release the annotated data extracted from the $I N$ SPIRE platform, on which our experiments are based. To the best of our knowledge, data of this size and coverage is the first to be available in author disambiguation research. By releasing the data publicly, we hope to provide the basis for further research on author disambiguation and related topics.

\section{References}

Breiman, L. (2001). Random forests. Machine learning, 45(1):5-32.

Buitinck, L., Louppe, G., Blondel, M., Pedregosa, F., Mueller, A., Grisel, O., Niculae, V., Prettenhofer, P., Gramfort, A., Grobler, J., Layton, R., VanderPlas, J., Joly, A., Holt, B., and Varoquaux, G. (2013). API design for machine learning software: experiences from the scikit-learn project. CoRR, abs/1309.0238.

Chin, W.-S., Zhuang, Y., Juan, Y.-C., Wu, F., Tung, H.-Y., Yu, T., Wang, J.-P., Chang, C.-X., Yang, C.-P., Chang, W.-C., et al. (2014). Effective string processing and matching for author disambiguation. The Journal of Machine Learning Research, 15(1):3037-3064.

Culotta, A., Kanani, P., Hall, R., Wick, M., and McCallum, A. (2007). Author disambiguation using error-driven machine learning with a ranking loss function. In Sixth International Workshop on Information Integration on the Web (IIWeb-07), Vancouver, Canada.

Fan, R.-E., Chang, K.-W., Hsieh, C.-J., Wang, X.-R., and Lin, C.-J. (2008). Liblinear: A library for large linear classification. The Journal of Machine Learning Research, 9:18711874.

Fan, X., Wang, J., Pu, X., Zhou, L., and Lv, B. (2011). On graph-based name disambiguation. Journal of Data and Information Quality (JDIQ), 2(2):10. 
Fellegi, I. P. and Sunter, A. B. (1969). A theory for record linkage. Journal of the American Statistical Association, 64:1183-1210.

Ferreira, A. A., Gonçalves, M. A., and Laender, A. H. (2012). A brief survey of automatic methods for author name disambiguation. Acm Sigmod Record, 41(2):15-26.

Ferreira, A. A., Veloso, A., Gonçalves, M. A., and Laender, A. H. (2010). Effective selftraining author name disambiguation in scholarly digital libraries. In Proceedings of the 10th annual joint conference on Digital libraries, pages 39-48. ACM.

Friedman, J. H. (2001). Greedy function approximation: a gradient boosting machine. Annals of statistics, pages 1189-1232.

Gentil-Beccot, A., Mele, S., Holtkamp, A., O'Connell, H. B., and Brooks, T. C. (2009). Information resources in high-energy physics: Surveying the present landscape and charting the future course. Journal of the American Society for Information Science and Technology, 60(1):150-160.

Guyon, I., Weston, J., Barnhill, S., and Vapnik, V. (2002). Gene selection for cancer classification using support vector machines. Machine learning, 46(1-3):389-422.

Han, H., Giles, L., Zha, H., Li, C., and Tsioutsiouliklis, K. (2004). Two supervised learning approaches for name disambiguation in author citations. In Digital Libraries, 2004. Proceedings of the 2004 Joint ACM/IEEE Conference on, pages 296-305. IEEE.

Huang, J., Ertekin, S., and Giles, C. L. (2006). Efficient name disambiguation for large-scale databases. In Knowledge Discovery in Databases: PKDD 2006, pages 536-544. Springer.

Jones, E., Oliphant, T., Peterson, P., et al. (2001-). SciPy: Open source scientific tools for Python. [Online; accessed 2015-08-10].

Kang, I.-S., Na, S.-H., Lee, S., Jung, H., Kim, P., Sung, W.-K., and Lee, J.-H. (2009). On co-authorship for author disambiguation. Information Processing 83 Management, $45(1): 84-97$.

Lange, D. and Naumann, F. (2011). Frequency-aware similarity measures: why arnold schwarzenegger is always a duplicate. In Proceedings of the 20th ACM international conference on Information and knowledge management, pages 243-248. ACM.

Levin, M., Krawczyk, S., Bethard, S., and Jurafsky, D. (2012). Citation-based bootstrapping for large-scale author disambiguation. Journal of the American Society for Information Science and Technology, 63(5):1030-1047.

Liu, W., Islamaj Doğan, R., Kim, S., Comeau, D. C., Kim, W., Yeganova, L., Lu, Z., and Wilbur, W. J. (2014). Author name disambiguation for pubmed. Journal of the Association for Information Science and Technology, 65(4):765-781.

Louppe, G., Wehenkel, L., Sutera, A., and Geurts, P. (2013). Understanding variable importances in forests of randomized trees. In Advances in Neural Information Processing Systems, pages 431-439.

Malin, B. (2005). Unsupervised name disambiguation via social network similarity. In Workshop on link analysis, counterterrorism, and security, volume 1401, pages 93-102.

McRae-Spencer, D. M. and Shadbolt, N. R. (2006). Also by the same author: Aktiveauthor, a citation graph approach to name disambiguation. In Proceedings of the 6th ACM/IEEECS joint conference on Digital libraries, pages 53-54. ACM.

Newman, M. E. (2001). The structure of scientific collaboration networks. Proceedings of the National Academy of Sciences, 98(2):404-409.

Pedregosa, F., Varoquaux, G., Gramfort, A., Michel, V., Thirion, B., Grisel, O., Blondel, M., Prettenhofer, P., Weiss, R., Dubourg, V., Vanderplas, J., Passos, A., Cournapeau, D., Brucher, M., Perrot, M., and Duchesnay, E. (2011). Scikit-learn: Machine learning in Python. Journal of Machine Learning Research, 12:2825-2830.

Philips, L. (2000). The double metaphone search algorithm. C/C++ Users J., 18(6):38-43.

Ruggles, S., Sobek, M., Fitch, C. A., Hall, P. K., and Ronnander, C. (2008). Integrated public use microdata series. Historical Census Projects, Department of History, University of Minnesota. 
Schulz, C., Mazloumian, A., Petersen, A. M., Penner, O., and Helbing, D. (2014). Exploiting citation networks for large-scale author name disambiguation. EPJ Data Science, 3(1):114.

Smalheiser, N. R. and Torvik, V. I. (2009). Author name disambiguation. Annual review of information science and technology, 43(1):1-43.

Soler, J. (2007). Separating the articles of authors with the same name. Scientometrics, $72(2): 281-290$.

Song, Y., Huang, J., Councill, I. G., Li, J., and Giles, C. L. (2007). Efficient topic-based unsupervised name disambiguation. In Proceedings of the 7th ACM/IEEE-CS joint conference on Digital libraries, pages 342-351. ACM.

Strotmann, A. and Zhao, D. (2012). Author name disambiguation: What difference does it make in author-based citation analysis? Journal of the American Society for Information Science and Technology, 63(9):1820-1833.

Taft, R. L. (1970). Name search techniques. Technical Report Special Report No. 1, New York State Identification and Intelligence System, Albany, NY.

The National Archives (2007). The soundex indexing system.

Torvik, V. I. and Smalheiser, N. R. (2009). Author name disambiguation in medline. ACM Transactions on Knowledge Discovery from Data (TKDD), 3(3):11.

Tran, H. N., Huynh, T., and Do, T. (2014). Author name disambiguation by using deep neural network. In Intelligent information and database systems, pages 123-132. Springer.

Treeratpituk, P. and Giles, C. L. (2009). Disambiguating authors in academic publications using random forests. In Proceedings of the 9th ACM/IEEE-CS joint conference on Digital libraries, pages 39-48. ACM.

Treeratpituk, P. and Giles, C. L. (2012). Name-ethnicity classification and ethnicity-sensitive name matching. In $A A A I$. Citeseer.

Ward Jr, J. H. (1963). Hierarchical grouping to optimize an objective function. Journal of the American statistical association, 58(301):236-244. 